\title{
A controlled respiratory training to improve quality of life and well-being perception in chronic obstructive pulmonary disease patients
}

Francesca Mastorci ${ }^{1}$, Francesca Mannucci ${ }^{2}$, Maria Francesca Lodovica Lazzeri ${ }^{1}$, Mirko Passera ${ }^{1}$, Fabio Brucini ${ }^{1}$, Ivana Pavlickova ${ }^{2}$, Alessandro Pingitore ${ }^{1 *}$ and Giosuè Catapano ${ }^{2}$

${ }^{1}$ Clinical Physiology Institute, CNR, Pisa, Italy,

${ }^{2}$ Fondazione G. Monasterio, Regione Toscana, Pisa, Italy

\begin{abstract}
Background: Chronic Obstructive Pulmonary Disease (COPD) requires continuous patient management caused by frequent exacerbations with consequent hospitalizations and a worsening of the patient's quality of life. Pulmonary rehabilitation improves exercise tolerance, but many patients do not have access to these programs.
\end{abstract}

Aim: We assumed that 3 months of controlled respiratory training (CRT), providing benefits on well-being in healthy subjects, can represent a valid strategy to improve health status and better disease management in COPD patients.

Design: Quality improvement in health care

Setting: Outpatients attending the rehabilitation treatment room of a single centre.

Population: 34 patients with COPD (according GOLD 2019 classification) were enrolled.

Methods: Patients were divided into TREAT group ( $\mathrm{n}=18,16$ males, age $73.83 \pm 6.71$ years) and control group (CTR: 13 males, age $74.06 \pm 6.38$ years). Before and after CRT a battery of psychological questionnaires were assessed. T

Results: REAT group in Post-CRT, as compared to CTR, showed a reduction in depressive symptoms (BDI, $\mathrm{p}<0.05$; POMS depression, $\mathrm{p}<0.05)$, anger ( $<<0.01$ ), stress perception (PSS, $\mathrm{p}<0.05$ ), and anxiety levels (BAI, $\mathrm{p}=0.05)$. Also, CRT improved general health $(\mathrm{p}<0.01)$ and vitality $(\mathrm{p}<0.05)$.

Conclusions: This preliminary study demonstrated that CRT is effective to improve quality of life and well-being perception in patients with COPD. Larger studies will need to confirm the long-term clinical and psychological benefits of this holistic approach, addictive to the traditional clinical practice, not only for symptomatic COPD, but also more generally to all patients with chronic diseases.

Clinical rehabilitation impact: In clinical settings, for COPD patients, who experience the chronic nature of illness, we propose an additive holistic approach to the traditional clinical practice to improve health-related quality of life, and thus, a better disease management.

\section{Introduction}

Chronic Obstructive Pulmonary Disease (COPD), require continuous patient management caused by frequent exacerbations with consequent hospitalizations and a worsening of the patient's quality of life [1]. Further, the clinical scenario of COPD is dominated by dyspnoea, that lead to a progressive inactivity, and thus, inducing progressive deconditioning and weakening of the skeletal muscles. This vicious circle contributes to a significant reduction in functional capacity up to the impossibility of sustaining the normal activities of daily life [2-4]. Furthermore, the difficulties related to the chronic condition can also decrease the perception of well-being and, facilitating the development of psychological comorbidities [5]. Among the management strategies of COPD, pulmonary rehabilitation is taking an increasingly central role that is underpinned by the American and European respiratory societies statement [6]. In this document, pulmonary rehabilitation is defined as a comprehensive and multidisciplinary intervention including exercise training, education, behaviour changes with the aim to improve physical activity and psychological conditions [6]. In this context, breathing exercises, including pursed lips breathing or inspiratory muscle training, as well as yoga techniques, have been shown to have a high level of effectiveness in reducing dyspnoea, and improving exercise tolerance and quality of life $[7,8]$.

Controlled respiratory training (CRT), a method of respiratory training based on Pranayama Yoga procedure, is characterized by conscious slow and deep acts of breathing, without any device support [9]. This method provides benefits on well-being in healthy subjects

${ }^{\star}$ Correspondence to: Alessandro Pingitore, Clinical Physiology Institute, CNR, Via Moruzzi, Italy, E-mail: pingi@ifc.cnr.it

Key words: $C O P D$, controlled respiratory training, pulmonary rehabilitation, well being, quality of life

Received: February 03, 2021; Accepted: February 19, 2021; Published: February 23,2021 
and in patients with different diseases [10-12]. Therefore, we hypothesized that CRT could improve psychosocial dimensions in COPD patients. In line with this hypothesis, the aim of this pilot study was to assess the effects of CRT in COPD patients on quality of life and well-being perception.

\section{Materials and methods}

\section{Patients}

The enrolled population consisted of 18 patients ( 15 males) with COPD (according GOLD 2019 classification), evaluating their disease status according to FEV1 value ( $<$ or $\geq 50 \%$ predicted; C-D stage or A-B stage, respectively), presence of symptoms (MRC $\geq 2$, CAT $\geq 10$; B-D stage), history of exacerbations ( $<$ or $\geq 2$ in the previous year; A-B stage or C-D stage, respectively).

The control group (CTR, $\mathrm{n}=16$ ), including COPD patients did not participate in the respiratory training (waiting list). Exclusion criteria were: (1) bronchitic exacerbation of any degree (mild-moderatesevere) within 3 months prior to enrollment; (2) oxygen lung therapy; (3) general clinical contraindications to the cardiopulmonary exercise test according to the ATS/ACCP guidelines; (4) physical impediments to the procedure of the ergospirometry; (5) scares motivation to respiratory training. Data on medical history and clinical status were collected and summarized in Table 1. Medication did not change during the training period. The research protocol was approved by the local ethics committee review board (Prot. n 14372). All patients gave informed consent, and authorized physicians to use their clinical data in accordance with Italian law. All procedures performed in the study were in accordance with the ethical standards of the institutional and/ or national research committee and with the 1964 Helsinki declaration and its later amendments or comparable ethical standards.

\section{Intervention method}

CRT consisted of weekly $2 \mathrm{~h}$ sessions, lasting 3 months. This was divided into 2 parts: theoretical and practical. In the theoretical session, the educational sessions were the anatomy and physiology of the respiratory system, breathing control and breathing awareness, visualization. The practical part was performed indoors, paying attention to personal comfort and environmental quietness. The first step consisted of teaching the optimal position for correct breathing: the privileged position was the supine one. Patients were encouraged to slowly inhale, again through the nose, and bring the air into the bottom of their lungs with the diaphragm, then continue inhaling to open and expand the rib cage upward and finishing inhalation with clavicle respiration. The exhalation time is twice as long as the inhalation time: for example, when an inspiration lasts $4 \mathrm{~s}$, expiration lasts $8 \mathrm{~s}$. In the first step, the inspiration and expiration were repeated without a break, with nasal inhaling and oral exhaling, in order to control air flux. In the second step both air, inhalation, and exhalation, were accomplished nasally with breaks at maximal inspiration and at maximal expiration. These breaks lasted for two at the beginning and

Table 1. Demographic and clinical characteristics of the population. Data given as mean \pm SD. BMI: Body Mass Index; GOLD: Global Initiative for Chronic Obstructive Lung Disease; CAT: COPD Assessment Test.

\begin{tabular}{|l|c|c|c|}
\hline Variables & TREAT $(\mathbf{n}=\mathbf{1 8})$ & CTR $(\mathbf{N}=16)$ & p \\
\hline Age & $73.83 \pm 6.71$ & $74.06 \pm 6.38$ & $\mathrm{~ns}$ \\
\hline BMI $\left(\mathrm{Kg} / \mathrm{m}^{2}\right)$ & $25.4 \pm 3.22$ & $24.8 \pm 2.36$ & $\mathrm{~ns}$ \\
\hline Emphysema/ chronic bronchitis $(\mathrm{n})$ & $11 / 7$ & $10 / 6$ & $\mathrm{~ns}$ \\
\hline GOLD A/B/C/D & $5 / 7 / 2 / 4$ & $5 / 5 / 3 / 3$ & $\mathrm{~ns}$ \\
\hline MRC score 0/1/2 & $2 / 9 / 7$ & $1 / 11 / 5$ & $\mathrm{~ns}$ \\
\hline CAT score & $13.4 \pm 5.2$ & $11.5 \pm 3.8$ & $\mathrm{~ns}$ \\
\hline
\end{tabular}

up to eight seconds at the end of the training. These exercises gave patients a heightened awareness of their breathing thus enabling them to control both frequency and deepness. The control and the awareness of breathing were further enhanced using visualization techniques that are typical in yoga practice with the aim to increase concentration towards breathing. The weekly CRT sessions lasted $2 \mathrm{~h}$ each, for a total number of 14 sessions in the 3 months. The exercises during the three months increased in degree of difficulty by increasing the in/out times and therefore decreasing the acts per minute, but always respecting the comfort of the patients. Patients were invited to perform CRT exercises at home with the aid of an audio for the ventilatory rhythm and were regularly encouraged by our trainers through mobile-phone messages and/or whatsapp communication.

\section{Measures}

The study included measurements in baseline condition, prior to randomization (Pre-CRT) and after 3 months of CRT (Post-CRT). During this time, all participants were advised to do not modify their usual lifestyle. At baseline and at the end of training period, all patients were submitted to a selection of psychometric tests specifically designed for evaluating psychosocial profile. In particular, the quality and patterns of sleep (Pittsburgh Sleep Quality Index; PSQI) [13], affective dimension (Profile of Mood States; POMS, Beck Depression Inventory; BDI-II, Beck Anxiety Inventory; BAI) [14-15], stress perception (Perceived Stress Scale; PSS) [16], and well-being (Psychological General Well-Being Index; PGWBI) were assessed [17].

\section{Statistical analysis}

Statistical data analyses were performed using SPSS (Version 22.0. Armonk, NY: IBM Corp). Data are presented as mean \pm SD or as mean with $95 \%$ confidence interval (CI). The Saphiro-Wilk test was used to assess the normality of data distribution. A p value $\leq 0.05$ was considered statistically significant. Changes in psychosocial profile between groups (Pre-CRT vs Post-CRT) were analyzed by Student's unpaired t-test, while variations intra group (Pre-CRT vs Post-CRT) with Student's paired t-test.

\section{Results}

\section{Patients demographic and clinical characteristics}

Demographic and clinical characteristics are shown in Table 1. Study population consisted of 34 male patients $(71 \pm 6.7$ years old $)$. All reported a history of smoking, with an index of tobacco exposure of $44 \pm 25$ pack years (range 10-90). The $65 \%$ of patients presented cardiovascular comorbidities, $12 \%$ suffering of diabetes mellitus, $6 \%$ from chronic renal insufficiency, while no one reported previous specific pulmonary episodes. All patients had COPD according to the GOLD classification and $65 \%$ of them was classified as stage B-D.

\section{Exercise program adherence}

During the entire duration of the CRT, the patients were clinically stable, maintained the prescribed therapy and did not need any type of additional intervention or therapy. The number of training sessions over 3 months were 24 . All patients performed training at home with a frequency of $19.17 \pm 2.9 \mathrm{~min}$ a day.

\section{Effect of respiratory training on quality of life and psychophysical well-being perception}

Descriptive data of psychometric questionnaires in all patients divided by group are presented in Table 2 . All variables were normally 
Table 2. Psychological variables in both groups $n$ baseline conditions (Pre-CRT) and after 3-months respiratory training (Post-CRT). Data given as mean \pm SD. BDI: Beck Depression Inventory; PSS: Perceived Stress Scale; BAI: Beck Anxiety Inventory; PSQI: Pittsburgh Sleep Quality Index; POMS: Profile of Mood States; PGWBI: Psychological General Well-Being Index; GWBI: General Well-Being Index; $\boldsymbol{p}^{\S}$ TREAT Pre-CRT vs TREAT Post-CRT; $\boldsymbol{p}^{\sharp}$ CTR Pre-CRT vs CTR Post-CRT.

\begin{tabular}{|c|c|c|c|c|c|c|c|c|c|}
\hline & \multirow[t]{2}{*}{ Variables } & \multicolumn{2}{|c|}{ Pre-CRT } & \multirow[b]{2}{*}{$p$} & \multicolumn{2}{|c|}{ Post-CRT } & \multirow[b]{2}{*}{$p$} & \multirow[b]{2}{*}{$p^{\S}$} & \multirow[b]{2}{*}{$p^{\#}$} \\
\hline & & TREAT $(n=18)$ & $\begin{array}{c}\text { CTR } \\
(n=16)\end{array}$ & & TREAT $(n=18)$ & $\begin{array}{c}\text { CTR } \\
(n=16)\end{array}$ & & & \\
\hline & BDI & $11.5 \pm 6.77$ & $11.31 \pm 7.19$ & $=0.84$ & $6.06 \pm 5.98$ & $11.18 \pm 7.26$ & $<0.05$ & $<0.001$ & $=0.33$ \\
\hline & PSS & $16.94 \pm 5.91$ & $16.31 \pm 5.62$ & $=0.75$ & $10.56 \pm 4.64$ & $16.06 \pm 5.55$ & $<0.05$ & $<0.001$ & $=0.16$ \\
\hline & BAI & $9.11 \pm 5.36$ & $8.94 \pm 5.22$ & $=0.92$ & $5 \pm 4.23$ & $8.56 \pm 5.66$ & $=0.05$ & $<0.001$ & $=0.14$ \\
\hline & PSQI & $5.83 \pm 3.49$ & $6.31 \pm 3.11$ & $=0.68$ & $5.83 \pm 3.79$ & $6.69 \pm 2.36$ & $=0.44$ & $=1$ & $=0.3$ \\
\hline \multirow{7}{*}{$\sum_{0}^{\infty}$} & Tension & $45.28 \pm 7.91$ & $45.88 \pm 8.02$ & $=0.83$ & $44 \pm 7.78$ & $46.06 \pm 6.89$ & $=0.42$ & $=0.31$ & $=0.67$ \\
\hline & Depression & $52.00 \pm 9.45$ & $49.06 \pm 9.74$ & $=0.38$ & $43.28 \pm 5.11$ & $49.06 \pm 9.50$ & $<0.05$ & $<0.001$ & $=1$ \\
\hline & Anger & $49.39 \pm 7.83$ & $48.06 \pm 8.93$ & $=0.65$ & $41.22 \pm 6.84$ & $48.25 \pm 9.14$ & $<0.01$ & $<0.001$ & $=0.38$ \\
\hline & Vigor & $48.72 \pm 11.53$ & $47.88 \pm 11.79$ & $=0.83$ & $52.22 \pm 12.06$ & $47.75 \pm 11.76$ & $=0.35$ & $=0.1$ & $=0.58$ \\
\hline & Fatigue & $47.89 \pm 10.78$ & $48.81 \pm 10.63$ & $=0.8$ & $47.44 \pm 10.23$ & $49 \pm 10.51$ & $=0.61$ & $=0.75$ & $=0.33$ \\
\hline & Confusion & $45.44 \pm 8.00$ & $46.44 \pm 8.59$ & $=0.73$ & $44.56 \pm 7.78$ & $46.75 \pm 8.9$ & $=0.41$ & $=0.12$ & $=0.33$ \\
\hline & Total score & $240 \pm 38.94$ & $238.25 \pm 41.46$ & $=0.9$ & $220.5 \pm 32.88$ & $239.13 \pm 40.48$ & $=0.13$ & $<0.001$ & $=0.21$ \\
\hline \multirow{7}{*}{$\sum_{0}^{\infty}$} & Anxiety & $18 \pm 4.07$ & $17.63 \pm 4.30$ & $=0.8$ & $18.56 \pm 2.23$ & $17.38 \pm 4.10$ & $=0.3$ & $=0.51$ & $=0.52$ \\
\hline & Depressed mood & $9.94 \pm 0.94$ & $9.44 \pm 1.36$ & $=0.21$ & $10 \pm 0.97$ & $9.69 \pm 1.54$ & $=0.48$ & $=0.83$ & $=0.47$ \\
\hline & Positive well-being & $10.94 \pm 1.73$ & $10.81 \pm 1.47$ & $=0.81$ & $10.67 \pm 1.24$ & $10.69 \pm 1.54$ & 00.97 & $=0.62$ & $=0.54$ \\
\hline & Self-control & $6 \pm 1.41$ & $6.13 \pm 1.45$ & $=0.8$ & $6 \pm 1.28$ & $6.38 \pm 1.15$ & $=0.38$ & $=1$ & $=0.47$ \\
\hline & General health & $7.67 \pm 1.75$ & $7.44 \pm 1.50$ & $=0.69$ & $9.61 \pm 2.57$ & $7.31 \pm 1.99$ & $<0.001$ & $<0.001$ & $=0.61$ \\
\hline & Vitality & $7 \pm 1.91$ & $7.81 \pm 1.33$ & $=0.16$ & $10.50 \pm 1.89$ & $8.63 \pm 2.31$ & $<0.05$ & $<0.001$ & $=1$ \\
\hline & GWBI & $59.56 \pm 5.39$ & $59.25 \pm 5.78$ & $=0.87$ & $65.33 \pm 3.5$ & $60.06 \pm 6.31$ & $<0.01$ & $=0.43$ & $=1$ \\
\hline
\end{tabular}

distributed according to the Saphiro-Wilk test. At baseline conditions (Pre-CRT), the two groups showed no differences for any of the variables analyzed (Table 2). Regarding Post-CRT, several variables significantly differed between the two groups. COPD patients after CRT showed a reduction in depressive symptoms (BDI, $\mathrm{p}<0.05)$, as compared to control counterpart. CRT, in treated patients, reduced POMS depression $(\mathrm{p}<0.05)$ and POMS anger $(\mathrm{p}<0.01)$, stress perception (PSS, $\mathrm{p}<0.05$ ), and anxiety levels (BAI, $\mathrm{p}=0.05)$. CRT improved psychological general well-being index in terms of general health $(\mathrm{p}<0.01)$ and vitality $(\mathrm{p}<0.05)$. No differences between groups were found in sleep quality.

Intra-group analysis has revealed that CRT patients in Post-CRT phase had a better perception of their quality of life and well-being. In fact, while control population did not change any variable compared to the baseline assessment (Table 2), CRT group showed no depressive symptoms (BDI, $\mathrm{p}<0.001$ and POMS depression, $\mathrm{p}<0.001)$ and aggressive behavior (POMS anger, $\mathrm{p}<0.001$ ), a reduction in stress (PSS, $\mathrm{p}<0.001$ ), and anxiety perception (BAI, $\mathrm{p}<0.001$ ), and an enhanced well-being level (PWBI general health $\mathrm{p}<0.001$, and PWBI vitality, $\mathrm{p}<0.001)$.

\section{Discussion}

The present study explores the impact of CRT on quality of life and well-being perception in COPD patients, demonstrating that this approach is associated with improved psychological profile. More in detail, the main results of this pilot study showed that patients with symptomatic COPD had a significantly better well-being perception in terms of reduction in depressive symptoms, anxiety and stress levels, and enhanced general health awareness after CRT compared with the control group.

Our rationale for using controlled respiratory training is that this is a complementary intervention that is focused mainly on the cure of the person and for this reason has to be considered in addition to the state of the art treatment. Moreover, the approach here proposed is simple, cheaper, and can be also performed at home by the patients after a dedicated and specific training period. Accordingly, in our study, the rate of CRT home-training was high, that is about 20 minutes a day. This is an important result if we consider that in COPD patients, adherence to any kind of treatment, both pharmacological or not, is low $[18,19]$. In addition, the beneficial effects of pulmonary rehabilitation diminish with time and lack of compliance due to subjective factors, including smoking status, severity of dyspnoea, anxiety and depression, length of program, frequency of hospitalizations, and the difficulties to access to these care protocols [20-22]

This highlights the need for the improvement, also through disease complementary interventional approaches [23]. In this line, previous studies reported that breathing exercises could reduce anxiety and depression, and anger and confusion [24]. More generally, Pranayama techniques are able to induce relaxation and increase attention levels [25]. From clinical perspective, a recent study has demonstrated in our target patient population, that pranayama, or yoga breathing, was associated with improved exercise tolerance. ${ }^{26}$ However, in addition to the clinical aspects, it is also useful to underpin that emotional and social wellbeing are involved in COPD management. As the disease progresses, it becomes more difficult to carry out even the simplest daily activities, so much so that only $30.6 \%$ of COPD patients say they are satisfied with their life [27]. According to this view, our data demonstrated that three-month, twice-weekly of CRT, as compared to baseline condition was effective in reducing perception of anxiety, depression and stress levels and increasing well-being and vitality in COPD patients. Our preliminary results make this approach applicable in the primary care clinic in COPD field during pulmonary rehabilitation programs, but mostly at home, inasmuch our methodology could be used for patients who do not have access to a formal program of pulmonary rehabilitation or even be in support of the traditional practice. All this leads us to highlight even more the need to propose a more "holistic" approach in this category of patient and more generally in patients suffering from chronic diseases. In fact, when we talk about chronic diseases, we are talking about diseases that accompany the patient throughout his or her life. A patient with a chronic illness becomes a person with a need of much more attention and care, who over time is forced to limit his or her daily activities and social relationships. In this condition, the 
terms 'to cure' and 'to care' must be drawn to attention. In line with Humanistic Medicine patient-centred view, taking care of a patient no longer means merely treating the illness by managing the symptoms, but means taking care of the person in entirety: treating the illness, but also assessing how he or she lives with it, his or her psychological sphere and his or her social support. In addition, CRT here described is already applied by our research group in adolescent Fontan patients demonstrating that this methodology increases significantly aerobic capacities, and, thus, quality of life [9].

Finally, it is possible that the practice of respiratory training made patients feel better and, therefore, more likely to engage in daily activities, contributing to their improved quality of life, unrelatedly of an actual improvement in clinical parameters.

\section{Limitations of the study}

First, the small sample of the population study reduces the robustness of the results. However, the sample was homogeneous for age and clinical characterization. Second, although the social component is very important in COPD patients, the "group" factor was not investigated, and this aspect could be evaluated in future studies.

\section{Conclusion}

Our findings demonstrate that practice of CRT by patients with COPD, based on diaphragmatic respiration, is feasible, well tolerated and effective to improve quality of life and well-being perception in the short term. In COPD patients, adherence to treatment for both breathing and other concomitant medical conditions is lower than in other chronic diseases. However, the patient, due to the chronic nature of his illness, is called to be an active and collaborative agent within the treatment process especially because this chronic condition, in its evolution and prognosis, may vary and often it is accompanied by comorbidity with other diseases or psychological disorders.

This highlights the need for the improvement, also through disease non-traditional programs [23]. Furthermore, despite previous studies have shown that pulmonary rehabilitation programs improve exercise tolerance and improve health-related quality of life, this traditional approach is not always available to all patients with COPD, for high costs and other concomitants factors such as presence of anxiety and depression [22].

CRT, here proposed, appears an additive holistic approach to the traditional clinical practice. However, larger studies will need to confirm these preliminary results, also taking into account long-term psychosocial benefits associated to CRT.

\section{Acknowledgements}

The authors would like to acknowledge the cooperation of the nursing and medical staff in the study hospital and COPD patients.

\section{References}

1. Barnes PJ, Celli BR (2009) Systemic manifestations and comorbidities of COPD. Eur Respir J 33: 1165-1185. [Crossref]

2. Celli B, Cote CG, Marin JM, Casanova C, Montes de Oca M, et al. (2004) The BodyMass Index, Airflow Obstruction,Dyspnea, and Exercise Capacity Index in Chronic Obstructive Pulmonary Disease. N Engl J Med 350: 1005-1012. [Crossref]

3. Mannino DM, Buist AS (2007) Global burden of COPD: risk factors, prevalence, and future trends. Lancet 370: 765-773. [Crossref]

4. Soriano JB, Zielinski J, Price D (2009) Screening for and early detection of chronic obstructive pulmonary disease. Lancet 374: 721-732. [Crossref]

5. Zareifopoulos N, Bellou A, Spiropoulou A, Spiropoulos K (2019) Prevalence, Contribution to Disease Burden and Management of Comorbid Depression and Anxiety in Chronic Obstructive Pulmonary Disease: A Narrative Review. COPD 16: 406-417. [Crossref]
6. Spruit MA, Singh SJ, Garvey C, ZuWallack R, Nici L, et al. (2013) An official American Thoracic Society/European Respiratory Society Statement: key concepts and advances in pulmonary rehabilitation. Am J Respir Crit Care Med 188: e13-64. [Crossref]

7. Cote CG, Celli BR (2005) Pulmonary rehabilitation and the BODE index in COPD. Eur Respir J 26: 630-636. [Crossref]

8. Ochmann U, Jörres RA, Nowak RA (2012) Long-term Efficacy of Pulmonary Rehabilitation: A STATE-OF-THE-ART REVIEW. J Cardiopulm Rehabil Prev 32: 117-1126. [Crossref]

9. Ait Ali L, Pingitore A, Piaggi P, Brucini F, Passera M, et al. (2018) Respiratory Training Late After Fontan Intervention: Impact on Cardiorespiratory Performance. Pediatr Cardiol 39: 695-704. [Crossref]

10. Bernardi L, Passino C, Spadacini G, Bonfichi M, ArcainiL, et al. (2007) Reduced hypoxic ventilatory response with preserved blood oxygenation in yoga trainees and Himalayan Buddhist monks at altitude: evidence of a different adaptive strategy? Eur J Appl Physiol 99: 511-518. [Crossref]

11. Brown RP, Gerbarg PL (2009) Yoga breathing, meditation, and longevity. Ann NY Acad Sci. 1172: 54-62. [Crossref]

12. Paul-Labrador M, Polk D, Dwyer JH, Velasquez I, Nidich S, et al. (2006) Effects of a randomized controlled trial of transcendental meditation on components of the metabolic syndrome in subjects with coronary heart disease. Arch Int Med 166: 1218 1224. [Crossref]

13. Conti L (2000) Directory of Assessment Scales in Psychiatry. Progress in Psychiatry series. SEE Florence: 3.

14. Sanavio E, Sica C (1999) Personality tests. Inventories and questionnaires. The mill.

15. Julian LJ (2011) Measures of anxiety: State-Trait Anxiety Inventory (STAI), Beck Anxiety Inventory (BAI), and Hospital Anxiety and Depression Scale-Anxiety (HADS-A). Arthritis Care Res (Hoboken) 63: S467-S72. [Crossref]

16. Nielsen MG, Ørnbøl E, Vestergaard M, Bech P, Larsen FB, et al. (2016) The construct validity of the Perceived Stress Scale. J Psychosom Res 84: 22-30. [Crossref]

17. Hopton JL, Hunt SM, Shiels C, Smith C (1995) Measuring psychological well-being The adapted General Well-Being Index in a primary care setting: a test of validity. Fam Pract 12: 452-460. [Crossref]

18. Sanduzzi A, Balbo P, Candoli P, Catapano GA, Contini P, et al. (2104) COPD: adherence to therapy. Multidiscip Respir Med 9: 60. [Crossref]

19. Scalone G, Nava S, Ventrella F, Bussoli G, Catapano GA, et al. (2018) Pharmacologica approach and adherence to treatment recommendations in frequently and nonfrequently exacerbating COPD patients from Italy: MISTRAL - The prospective cohort, observational study. Pulm Pharmacol Ther 53: 68-77. [Crossref]

20. Fan VS, Giardino ND, Blough DK, Kaplan RM, Ramsey SD, et al. (2008) Costs of pulmonary rehabilitation and predictors of adherence in the National Emphysema Treatment Trial. COPD 5: 105-116. [Crossref]

21. Sabit R, Griffiths TL, Watkins AJ, Evans W, Bolton CE, et al. (2008) Predictors of poor attendance at an outpatient pulmonary rehabilitation programme. Respir Med 102 : 819-824. [Crossref]

22. van Gestel AJ, Kohler M, Steier J, Teschler S, Russi EW, et al. (2012) The effects of controlled breathing during pulmonary rehabilitation in patients with COPD. Respiration 83: 115-124. [Crossref]

23. Königsdorfer N, Jörres RA, Söhler S, Welte T, Behr J, et al. (2009) Perceived Health Status as Measured by the SF-36 in Patients with Multiple Sclerosis: A Review. Scand J Caring Sci 23: 529-538. [Crossref]

24. Fumoto M, Sato-Suzuki I, Seki Y, Mohri Y, Arita H (2004) Appearance of highfrequency alpha band with disappearance of low-frequency alpha band in EEG is produced during voluntary abdominal breathing in an eyes-closed condition. Neurosci Res 50: 307-317. [Crossref]

25. Zaccaro A, Piarulli A, Laurino M, Garbella E, Menicucci D, et al. (2018) How Breath-Control Can Change Your Life: A Systematic Review on Psycho-Physiological Correlates of Slow Breathing. Front Hum Neurosci 12: 353. [Crossref]

26. Kaminsky DA, Guntupalli KK, Lippmann J, Burns SM, Brock MA, et al. (2017) Effect of Yoga Breathing (Pranayama) on Exercise Tolerance in Patients with Chronic Obstructive Pulmonary Disease: A Randomized, Controlled Trial. J Altern Complement Med 23: 696-704. [Crossref]

27. Jang SM, Kim KU, Na HJ, Song SE, Lee SH, et al. (2019) Depression is a major determinant of both disease-specific and generic health-related quality of life in people with severe COPD. Chron Respir Dis 16: 1479972318775422. [Crossref]

Copyright: (C)2021 Mastorci F. This is an open-access article distributed under the terms of the Creative Commons Attribution License, which permits unrestricted use, distribution, and reproduction in any medium, provided the original author and source are credited. 